\title{
Retinoic acid decreases targeting of p27 for degradation via an N-myc-dependent decrease in p27 phosphorylation and an $\mathrm{N}$-myc-independent decrease in Skp2
}

\author{
M Nakamura ${ }^{1}$, T Matsuo ${ }^{1}$, J Stauffer ${ }^{1}$, L Neckers ${ }^{2}$ and CJ Thiele ${ }^{*, 1}$ \\ ${ }^{1}$ Cell and Molecular Biology Section, Pediatric Oncology Branch, Center for \\ Cancer Research, National Cancer Institute, Bldg. 10 Rm 13N240, 10 Center \\ Drive MSC-1928, Bethesda, MD 20892, USA \\ 2 Cell and Cancer Biology Branch, Center for Cancer Research, National Cancer \\ Institute, 9610 Medical Center Drive, Suite 300, Rockville, MD 20850, USA \\ * Corresponding author: CJ Thiele, Tel: +1-301-496-1543; \\ Fax: +1-301-402-0575; E-mail: ct47a@nih.gov
}

Received 15.5.02; revised 5.8.02; accepted 5.8.02

Edited by G Melino

\begin{abstract}
Poor prognosis neuroblastoma (NB) tumors are marked by amplification and overexpression of $\mathrm{N}$-myc. Retinoic acid (RA) decreases $\mathrm{N}$-myc levels and induces cell cycle arrest in vitro and increases event-free survival in advanced stage NB patients. In this study, we investigated the mechanism(s) by which RA regulates cell cycle and how $\mathrm{N}$-myc affects NB cell cycle progression. Constitutive $\mathrm{N}$-myc overexpression stimulates increases in cyclin E-dependent kinase activity and decreases in p27 resulting in increased DNA synthesis. $\mathrm{N}$ myc regulates p27 levels through an increase in targeting of p27 to the proteasome via cyclin E kinase-dependent phosphorylation of p27 and its ubiquitination. N-myc also stimulates an increase in proteasome activity. In RA-treated cells in which $\mathrm{N}$-myc levels decline as p27 levels increase, degradation of p27 is also decreased. However, RA does not affect the activity of proteasome. The decrease in the degradation of p27 in RA-treated cells is due in part to a decrease in the N-myc stimulated phosphorylation of p27. However, RA also decreases Skp2 levels thus impairing the ability of p27 to be ubiquitinated. Thus, RA induces both Nmyc-dependent and -independent mechanisms to minimize the degradation of p27 and arrest NB cell growth.

Cell Death and Differentiation (2003) 10, 230-239. doi:10.1038/ sj.cdd. 4401125
\end{abstract}

Keywords: N-my c; retinoics; p27 neuroblastoma; SKP2; CDK6; proteosome; ubiquitination

Abbreviations: Cdk, cyclin-dependent kinase; Rb, retinoblastoma gene; RA, all-trans-retinoic acid; NB, neuroblastoma; BrdU, bromodeoxyuridine; HRP, horseradish peroxidase; LC, lactacystin.

\section{Introduction}

Neuroblastoma (NB), tumors of embryonal neural crest cells, is the most frequent extracranial solid tumors in children. NB has a high rate of spontaneous regression and differentiation to benign ganglioneuroma in vivo. ${ }^{1}$ However, NB tumors that have the worst prognosis are marked by amplification and overexpression of $\mathrm{N}$-myc. ${ }^{2}$ For this reason, drugs that are able to induce differentiation in NB or regulate N-myc levels have been studied in vitro as possible therapeutics. Retinoids are known to decrease $\mathrm{N}$-myc levels and cell proliferation and induce differentiation of NB cell lines in vitro. ${ }^{3}$ A recent clinical study has shown that retinoic acid (RA) improves event-free survival in NB patients with minimal residual disease after intensive chemotherapy. ${ }^{4}$ Although the biological effects of RA on NB differentiation have been studied, ${ }^{3,5}$ the mechanisms by which RA induces an arrest of NB cell cycle have not been well defined.

Previously, we demonstrated that RA induced a decrease in $\mathrm{N}$-myc mRNA and protein levels and an increase in p27 levels in growth arrested NB cells. ${ }^{6}$ This led us to hypothesize that these were important components in the ability of RA to arrest NB cell growth and raised the possibility that N-myc may regulate p27 levels. Studies on another member of the myc family indicate that c-myc acts upstream of Cdks in stimulating cell proliferation ${ }^{7}$ and increased c-myc expression in arrested fibroblasts results in the activation of Cdk activity and decreases in p27 levels. ${ }^{8}$ Furthermore, overexpression of cmyc abrogates p27-mediated cell cycle arrest by a mechanism in which $c-m y c$ sequesters $p 27^{9}$ by stimulating cyclin $D$ and the formation of cyclin D/Cdk4, 6 complexes that bind p27. ${ }^{10}$ Although some in vitro and in vivo studies indicate that $\mathrm{N}$-myc can replace $\mathrm{c}-m y c$, few studies have evaluated the effect of $\mathrm{N}$-myc on the cell cycle. ${ }^{11}$ During development c-myc is frequently found in tissues with high proliferative potential while $\mathrm{N}$-myc is more highly expressed in a tissue-restricted manner in the kidney, brain, B lymphocytes and hair follicles and in cells undergoing terminal differentiation. Thus the effect of $\mathrm{N}$-myc on the cell cycle in the context of terminal differentiation may differ from the effects of $c$-myc on cell cycle.

$\mathrm{N}$-myc overexpression has been shown to shorten the $\mathrm{G} 1$ phase of the NB cell cycle ${ }^{12}$ and induce the re-entry of quiescent cells into the cell cycle. ${ }^{13} \mathrm{RA}$ induces a decrease in $\mathrm{N}$-myc expression and $\mathrm{G} 1$ cell cycle arrest in NB cells ${ }^{3}$ and the constitutive expression of N-myc abrogates the RA-induced growth arrest and differentiation in NB cells. ${ }^{14}$ Recent evidence indicates that $\mathrm{N}$-myc inactivates the retinoblastoma gene $(\mathrm{Rb})$ path via induction of Id2. ${ }^{15} \mathrm{~N}$-myc is a well-known 
poor prognostic indicator in NB. A recent report indicates that a high level of p27 is a good prognostic factor in $\mathrm{NB}^{16}$ while decreased p27 is a poor prognostic factor in several malignant tumors. ${ }^{17}$

Cell cycle progression in eukaryotes is tightly regulated by cyclins and cyclin-dependent kinases (Cdks). ${ }^{18}$ cyclin/Cdks complexes are inhibited by cyclin-dependent kinase inhibitors (CKIs) including the Cip/Kip family (p21, p27, and p57), and the INK4 family (p15, p16, p18, and p19). While the Cip/Kip family members are considered potent inhibitors of cyclin E/ Cdk2 kinase, they may also act as positive regulators of cyclin D kinases. ${ }^{19}$ The cyclin D/Cdk4, 6 kinases are activated by mitogenic signals and these complexes contain Cip and Kip proteins. Sequestration of Cip/Kip protein to cyclin D/Cdk4, 6 complexes can activate cyclin E/Cdk2 kinases ${ }^{20}$ and these activated kinases phosphorylate $\mathrm{Rb}$, which releases E2F family proteins and stimulates the transcription of $S$ phase specific genes ${ }^{21}$ p27 levels are regulated negatively by mitogenic signals and positively by antimitogenic signals. ${ }^{22,23}$ The post-transcriptional regulation of p27 is mediated by proteolytic degradation of p27 levels by the ubiquitin-proteasome pathway. ${ }^{24,25}$ For the ubiquitination of p27, cyclin E/ Cdk2-dependent phosphorylation of p27 on threonine 187 is required. ${ }^{26}$ The phosphorylated p27 is specifically recognized by F-box protein Skp2 that is a component of SCF complex (Skp1, Cul1, F-box protein) for p27 and is subsequently degraded by ubiquitin-proteasome pathway. ${ }^{27}$

In this study, we sought to determine how N-myc affects the expression of cell cycle proteins in NB cells, specifically p27, and investigate the mechanism(s) regulating p27 levels in RAtreated G1-arrested NB cells. We find overexpression of $\mathrm{N}$ myc leads to increases in the expression of cyclin E, E2F, and an increase in the activity of cyclin E-dependent kinases and a subsequent phosphorylation of p27 and ubiquitination. We find that important components in the $R A$ regulation of proliferation in N-myc amplified NB cells are the increases in p27 that occur as a result of decreases in phosphorylation and ubiquitination of p27, which occur as a result of decreases in $\mathrm{N}$-myc and Skp2 expression.

\section{Results}

\section{Decreases in N-myc and Cdk6 and increases in p27 mark RA-induced G1 arrest in KCNR cells}

RA causes an arrest of cells at the $\mathrm{G} 1$ phase of the cell cycle and induces neuronal differentiation in NB cell lines. ${ }^{3,5} \mathrm{We}$ have shown that RA induced an accumulation of cells in G1 within $24-48$ h of RA treatment with more than $80 \%$ of cells in G1 by 120 h of RA treatment. To examine the effects of RA on regulation of cell cycle machinery, KCNR cells were treated with $5 \mu \mathrm{M}$ of RA for $72 \mathrm{~h}$ since the accumulation of cells in $\mathrm{G} 1$ reached a plateau after $72 \mathrm{~h}$ of $\mathrm{RA}$ treatment. ${ }^{6}$ After a $72 \mathrm{~h}$ RA treatment, flattening and disaggregation of cells was observed in RA-treated cultures of NB cells with cells showing neurite extensions (Figure 1a). The RA-induced differentiation is accompanied by inhibition of DNA synthesis, as a bromodeoxyuridine (BrdU) incorporation assay (Figure 1b) shows that incorporation of BrdU into DNA in RA-treated cells was significantly less than the control $(P<0.01)$. Furthermore, the accumulation of cells in G1 in RA-treated cells was significantly more than the control $(P<0.01)$. These results confirmed that RA inhibited DNA synthesis and induced a G1 arrest of cell cycle in NB cells.

Previously, we documented that RA induced a decrease in $\mathrm{N}$-myc and an increase in p27 with no significant changes in the levels of $\mathrm{G} 1$ cyclins, Cdk2 and Cdk4 proteins during the RA-induced G1 arrest of NB cells. ${ }^{6}$ In this study, we found that there was a five-fold decrease in the levels of Cdk6 protein in KCNR cells treated for $72 \mathrm{~h}$ with RA (Figure 1c). The steadystate levels of Cdk6 were decreased in time-dependent manner in RA-treated KCNR cells (data not shown). To determine whether $\mathrm{RA}$ regulates $\mathrm{p} 38^{\mathrm{Cdk} 6}$ levels at mRNA levels, Cdk6 mRNA expression was examined at various times after RA treatment (Figure 1d). Cdk6 mRNA levels decreased to $20 \%$ of control within $24 \mathrm{~h}$ of RA treatment with decreases observed as early as $5 \mathrm{~h}$ after retinoid treatment. Cdk2 and Cdk4 mRNA steady-state mRNA levels were unchanged during this time. These results indicate that Cdks are differentially regulated during retinoid treatment of NB cells.

The levels of CKIs were analyzed by Western blot analysis. RA induced increases in p27 levels, while p21 levels were unchanged (Figure 1c). KCNR cells do not express p15 and p16. ${ }^{6}$ Since the decrease in cyclin/Cdk-dependent kinase activity was coincident with an increase in p27 bound to G1 cyclin/Cdk in culture, we investigated whether RA could affect the G1 cyclin/Cdk kinase activity. The cyclin E-dependent kinase activities in cultures treated with RA were reduced to less than $20 \%$ of the control while cyclin D-dependent kinase activity was reduced to $50 \%$ of the control (Figure 1e). Consistent with our previous results ${ }^{6}$ the increases in p27 steady-state levels stimulated with RA were accompanied by increases in p27 bound to cyclin $E$ (Figure 1f). These results raise the possibility that decreases in N-myc and Cdk6 and an increase in p27 play important roles in RA induced arrest of NB cell growth.

\section{p27 levels and cell cycle regulation}

It is possible that the increase in $p 27^{\mathrm{kip} 1}$ levels may be a consequence and not the cause of the RA induced arrest of cells in G1. To examine this, KCNR cells were incubated in $0.5 \%$ serum for $48 \mathrm{~h}$, which caused a modest but reproducible increase in G1. Under these conditions Western blot analysis indicated that there was no change in either p27 or N-myc levels (Figure 2a). Cells were also incubated with the DNA polymerase inhibitor aphidicolin $(5 \mu \mathrm{g} / \mathrm{ml})$ in $0.5 \%$ serum for $48 \mathrm{~h}$. Aphidicolin arrests cells by blocking DNA replication and at a point after the RA restriction point (our unpublished data). Western blot analysis of lysates from these cells indicates that also under these conditions p27 levels do not increase and may even decrease in aphidicolin arrested cells compared to RA-treated cells (Figure 2a). Consistent with previous results, ${ }^{3}$ the levels of $\mathrm{N}$-myc do not decline to the same extent as in RA-treated cells.

While p27 was originally identified as an inhibitor of cyclin Edependent kinase ${ }^{20}$ it is also known to be an important component of the cyclin D kinase complex that stimulates cell

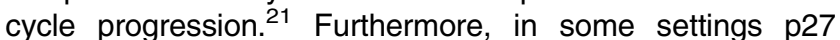
induces apoptosis while in others it is necessary for cell 
a

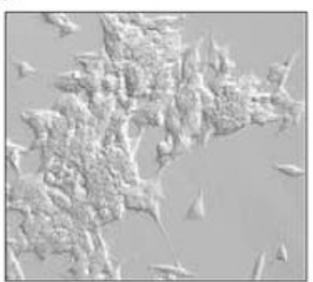

Control

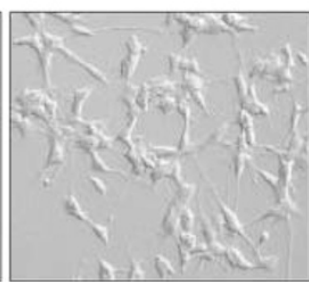

RA b

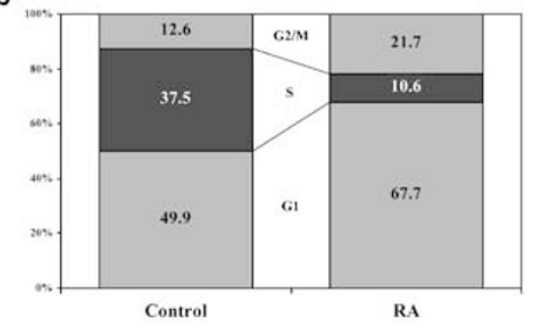

e

cyclin $\mathrm{E}$

kinase : $\mathrm{H} 1$

cyclin D

kinase : Rb
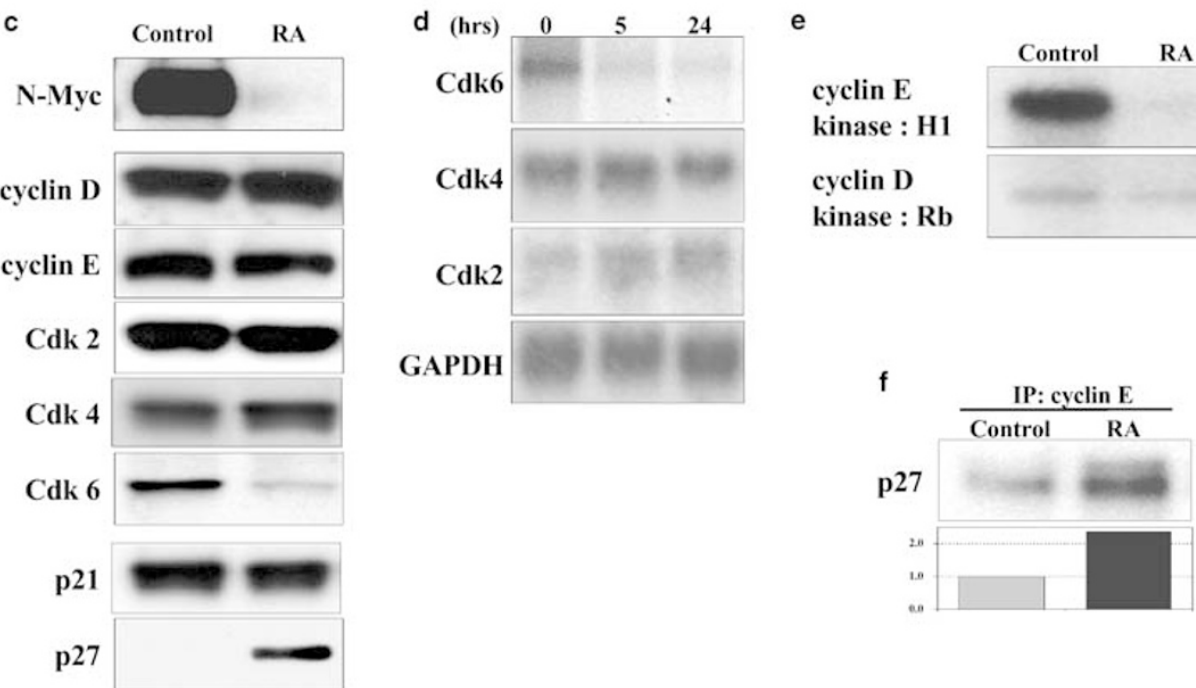

Figure 1 Decreases in N-myc and Cdk6 and increases in p27 mark RA-induced G1 arrest in KCNR cells. (a) RA induces neuronal differentiation in KCNR cells. KCNR cells were treated with solvent or $5 \mu \mathrm{M}$ RA for $72 \mathrm{~h}$. (b) RA decreases BrdU incorporation into DNA and arrest KCNR cells in G1. Flowcytometry data were analyzed by the CELL Quest (Becton Dickinson, San Jose, CA, USA). A representative cell cycle profile is shown and the accompanying graph represents 14 replicate cell cycle analyses taken at various times over the course of the experiments. For each cell cycle analysis the samples are run twice with less than $1 \%$ variation. Standard student's $t$-test was utilized to compare the values in the groups. (c) RA decreases N-myc and Cdk6 and increases p27. KCNR cells were treated with $5 \mu \mathrm{M}$ for $72 \mathrm{~h}$ and total proteins were analyzed by Western blot. (d) RA decreases Cdk6 mRNA. KCNR cells were treated with $5 \mu$ M RA for indicated time. (e) RA decreases cyclin E- and D-dependent kinase. Immunoprecipitations were performed with anti-cyclin E and anti-cyclin D1. Histone H1was used as substrate for cyclin E-kinase assay. GST-Rb fusion protein was used as substrate cyclin D-kinase assay. (f) RA decreases p27 bound to cyclin E. Cell extracts were immunoprecipitated with goat anti-cyclin E and immunoprecipitants were immunoblotted by rabbit anti-p27. The signals were quantified by densitometry and plot on the lower panel

survival. ${ }^{28}$ To examine the biologic effect of increased p27 expression in NB cells, KCNR cells were infected with an adenovirus containing the p27 gene. Infection with the p27 adenovirus increased p27 levels in a dose-dependent manner while null virus infections did not increase p27 levels (Figure 2b). As p27 levels increased, decreases in Cdk2 kinase activity were observed in p27 adenovirus infected cells. Furthermore, p27 adenovirus infection induced an increase in the accumulation of cells in $\mathrm{G} 1$ (at 5 and $10 \mathrm{MOI}$ ) compared to null virus; albeit this increase was lower than in cells treated with RA. There was no significant decrease in cell viability compared to null virus (data not shown). These results indicate that increases in p27 decrease DNA synthesis and cause an accumulation of NB cells in the G1 phase of the cell cycle.

\section{$\mathrm{N}$-myc regulation of $\mathrm{p} 27$}

Since RA decreases N-myc levels, increases p27 levels and arrests NB cells in $\mathrm{G}_{1}{ }^{3,6}$ we sought to examine the mechanism by which $\mathrm{N}$-myc affects proteins that regulate the cell cycle. The N-myc gene was transfected into SK-N-AS
(AS), which does not express N-myc. Stable G418-resistant $\mathrm{N}$-myc-expressing clones (1.6 and 14.2) were isolated as well as clones of SK-N-AS transfected with a control plasmid (6B and $8 \mathrm{~B}) .{ }^{29}$ In the $\mathrm{N}$-myc-expressing cells both protein and mRNA expression of $p 27$ were decreased compared to the levels in control-transfected cells (Figure 3a). Furthermore in $\mathrm{N}$-myc-expressing cells (AS1.6) there is a 3.1 -fold increase in cyclin $E$ levels and a 3.8-fold increase in cyclin E-dependent kinase activity compared to control-transfected cells (AS8B) (Figure 3b). The expression of E2F-1 mRNA was increased in $\mathrm{N}$-myc-expressing clones although there was not any significant change in the steady-state levels of E2F-1 protein (Figure $3 \mathrm{~b}$ ). These results indicate that $\mathrm{N}$-myc stimulates cyclin $E$ expression, its kinase activity and E2F-1 mRNA expression while inhibiting p27 expression. However, the levels of Cdk4 and Cdk6 were not changed in N-mycexpressing cells and cyclin D1 was decreased in N-mycexpressing cells compared to control cells (Figure $3 \mathrm{c}$ ). Thus there is a modest decrease in p27 mRNA and a marked decrease in p27 protein levels in N-myc-expressing cells and an increase in cyclin $E$, its kinase activation and E2F-1 levels. 
a

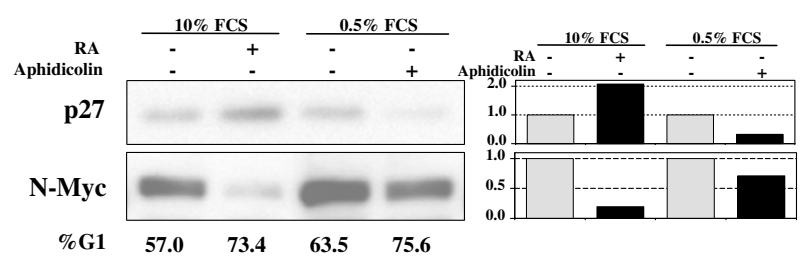

b

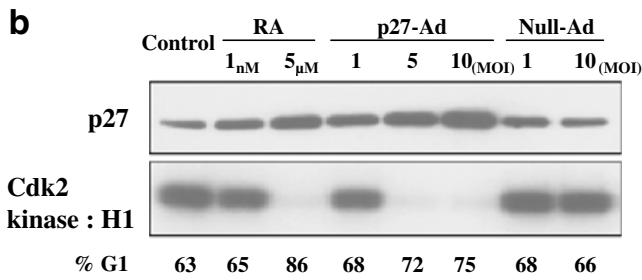

Figure 2 p27 levels and cell cycle regulation. (a) Aphidicolin-induced cell cycle arrest. KCNR cells were treated with $5 \mu \mathrm{g} / \mathrm{ml}$ aphidicolin in $0.5 \%$ serum for $48 \mathrm{~h}$ or with $5 \mu \mathrm{M} \mathrm{RA}$ for $48 \mathrm{~h}$. Protein extracts (20 $\mu \mathrm{g} / \mathrm{lane})$ were analyzed by Western blot for N-myc and p27. Quantification of protein expression is illustrated in the right panel. Protein expression of RA- or aphidicolin-treated cells is expressed relative to control expression. (b) p27 adenovirus infection inhibits Cdk2 kinase and arrest cell cycle. Protein extracts from KCNR cells treated with $1 \mathrm{nM}$ RA and infected with p27 or null adenovirus were analyzed by immunoblotting for p27 (upper panel). In vitro Cdk2-kinase assay. Immunoprecipitations by anti-Cdk2 were subjected for Cdk2-kinase assay using histone $\mathrm{H} 1$ as a substrate (lower panel) a

b

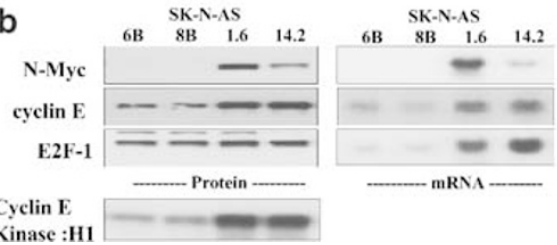

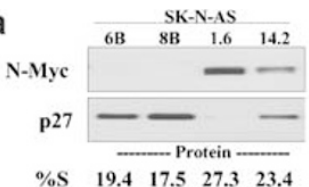
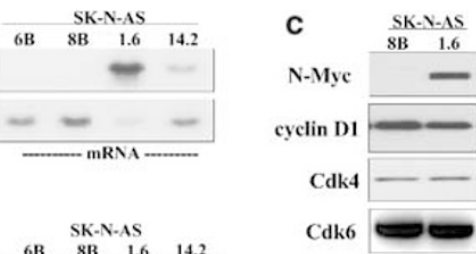

Figure $3 \mathrm{~N}$-myc regulation of p27. (a) N-myc overexpression inhibits p27. Protein extracts from AS1.6 and AS14.2 which are N-myc overexpressing cells, and AS6B and AS8B which are no-N-myc-expressing cells were analyzed by Western blot for N-myc and p27. Total RNA from same samples were analyzed by Northern blot for $\mathrm{N}$-myc and p27. (b) N-myc overexpression stimulates cyclin $\mathrm{E}$ and E2F. The same protein extracts were analyzed by Western blot analysis for cyclin E E2F-1. Immunoprecipitants by anti-cyclin E antibody were subjected to cyclin $\mathrm{E}$-kinase assay using histone $\mathrm{H} 1$ as a substrate. Total RNA were subjected for Northern blot analysis of cyclin E and E2F-1. (c) N-myc overexpression does not increase cyclin D/Cdk4, 6. Protein extracts from AS1.6 and AS8B were analyzed by Western blot for $\mathrm{N}$-myc and p27

\section{$\mathrm{N}$-myc affects p27 degradation by ubiquitin- proteasome pathway}

In this model system, the RA-induced increase in p27 occurred at 24-48 $\mathrm{h}$ coincident with the increase in cells in G1 and prior to the increase in p27 mRNA, which occurred at $96 \mathrm{~h} .{ }^{11}$ This indicated that post-transcriptional mechanisms most likely contribute to the increase in p27 during G1 arrest. p27 is regulated during the cell cycle via post-transcriptional mechanisms ${ }^{24}$ by proteolytic degradation of p27 levels via the ubiquitin-proteasome pathway. ${ }^{25}$ Skp1 and Cul1 are common proteins of the ubiquitin-protein ligase SCF complex, while Skp2 is the adapter subunit of SCF complex that specifically recognizes phosphorylated p27 and ubiquitinates it leading to its degradation by the proteasome. ${ }^{28}$

To examine whether the increased $\mathrm{N}$-myc expression affects the degradation of p27, His-tagged p27 was incubated with $\mathrm{S} 100$ cell extracts from $\mathrm{N}$-myc-expressing cells (AS1.6) and control-transfected cells (AS8B) for various times. Proteins were analyzed by SDS-PAGE and blotted with anti-His antibody. The His-tagged p27 was quantified by densitometry and analyzed by linear regression (StatView, Abacus Concepts Inc. CA, USA). The results indicated that the exogenous p27 is more rapidly degraded in lysates from $\mathrm{N}$-myc-expressing cells ( $50 \%$ degraded in $3.5 \mathrm{~h}$ ) compared to control-transfected cells (50\% degraded in $8.7 \mathrm{~h}$ ) (Figure $4 \mathrm{a}$ ).

At least, two steps are needed to degrade p27 by the ubiquitin-proteasome pathway, ubiquitination of p27 and degradation by $26 \mathrm{~S}$ proteasome. To examine the effect of $\mathrm{N}$-myc on proteasome activity in vitro, we measured the chymotoryptic activity of the proteasome by using a fluorogenic substrate, Suc-LLVY-AMC as described by Lightcap et al. ${ }^{30}$ The proteasome activity in $\mathrm{N}$-myc-expressing cells was significantly higher than in control-transfected cells. When we treated cells with $2 \mu \mathrm{M}$ lactacystin (LC) for $2 \mathrm{~h}$ prior to harvesting, the proteasome activity was negligible (Figure $4 b)$. To determine the effect of $\mathrm{N}$-myc on proteasome activity for p27 degradation in vivo, we treated AS8B and AS1.6 cells with $2 \mu \mathrm{M}$ of LC for $12 \mathrm{~h}$. LC increased p27 levels 4.3 -fold in AS8B cells and 4.8-fold in AS1.6 cells while it did not affect $N$ myc levels (Figure 4c). These results suggest that there is an increase in proteasome activity in $\mathrm{N}$-myc-expressing cells.

Proteasome degradation of p27 requires that p27 is first ubiquitinated. This ubiquitination step only occurs if p27 is phosphorylated. p27 is phosphorylated by cyclin E kinase and then is recognized specifically by Skp2 of the SCF complex (Skp1, Cul1, Skp2), which transfers activated ubiquitin to p27 by the ubiquitin-conjugating enzyme (E2). Recently, it has been shown that c-myc enhances Cul-1 levels and promotes G1 exit via Cul1-dependent ubiquitination and degradation of p27. ${ }^{28}$ We did not find that the steady-state levels of Cul1, Skp1 or Skp2 changed in N-myc-expressing cells (Figure 4d). Since cyclin E-dependent kinase phosphorylates p27 and phosphorylation of threonine-187 of p27 is required for its targeting for the ubiquitin-proteasome pathway, ${ }^{31}$ the increase in cyclin $\mathrm{E}$ kinase activity by $\mathrm{N}$-myc (Figure $3 \mathrm{~b}$ ) may lead to enhanced phosphorylation of p27. To examine whether the increased cyclin $\mathrm{E}$ kinase activity in N-mycexpressing cells can phosphorylate p27, we performed an in vitro kinase assay using recombinant p27 as a substrate. There is a 3.8-fold increase in phosphorylated recombinant p27 in the lysate from N-myc-expressing cells compared to the control lysate (Figure 4e). To assess whether this occurred in vivo, we examined the $\mathrm{N}$-myc-expressing cells (AS1.6) and control-transfected cells (AS8B) for the level of phosphorylated p27. p27 was immunoprecipitated from cell lysates using an anti-p27 antibody and immunoprecipitates were resolved by SDS-PAGE followed by immunoblotting with an antiphosphorylated p27 antibody. Even though the 
a

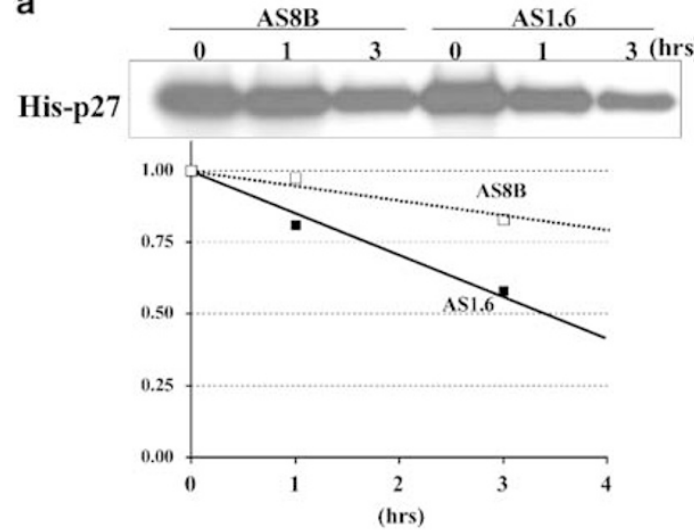

b

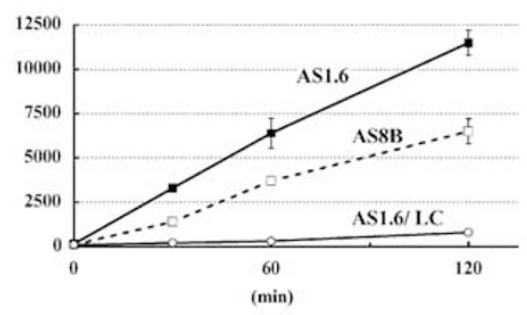

C

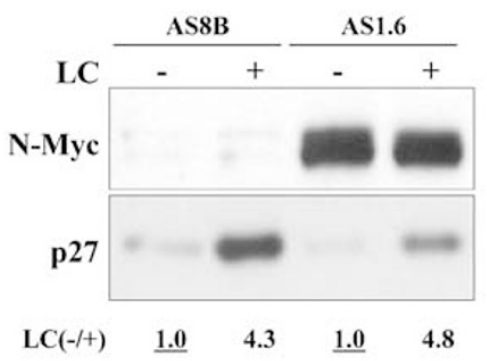

d

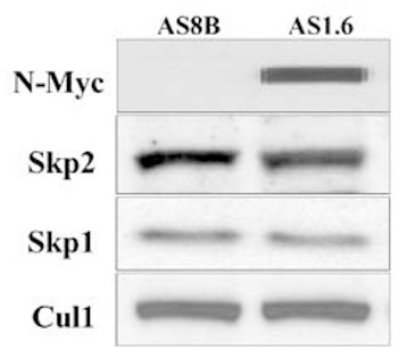

e

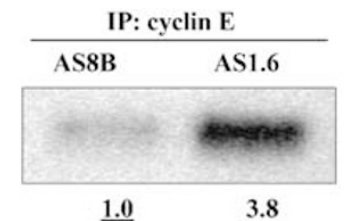

f
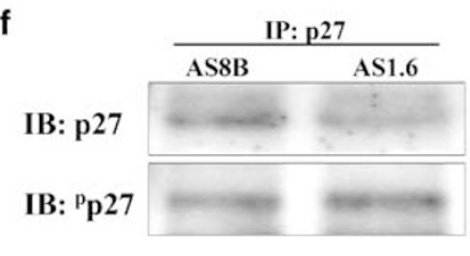

${ }^{\mathrm{p} p 27 / \mathrm{p} 27 \quad \underline{1.00}} \quad 1.49$

g

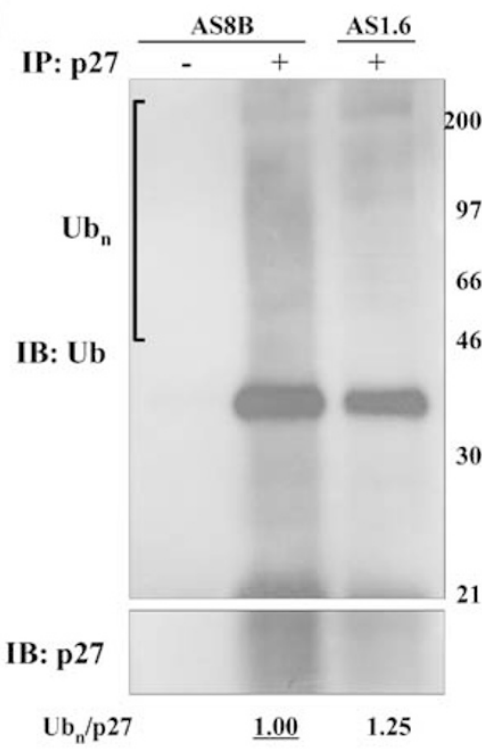

Figure $4 \mathrm{~N}$-myc affects p27 degradation by ubiquitin-proteasome pathway. (a) p27 in vitro degradation assay. His-tagged recombinant p27 were incubated with cell extracts (S100) from AS8B and AS1.6 cells for indicated time and his-tagged p27 was detected by immunoblotting using anti-His antibody. (b) N-myc overexpression increases proteasome activity. Total protein lysates from AS8B and AS1.6 were subjected for in vitro proteasome activity assay. To inhibit proteasome activity, AS1.6 were treated with $2 \mu \mathrm{M} \mathrm{LC}$ for $2 \mathrm{~h}$ before harvest. (c) Inhibition of proteasome increase p27 levels. AS8B and AS1.6 were treated with $2 \mu \mathrm{M} \mathrm{LC}$ for $12 \mathrm{~h}$ before harvest and total protein was subjected for Western blot analysis for N-myc and 27. (d) N-myc overexpression does not affect on SCF components. Protein lysate from AS8B and AS1.6 were analyzed by Western blot for Skp1, Cul1, and Skp2. (e) Increases in cyclin E kinase in N-myc-expressing cells (AS1.6) phosphorylates recombinant p27. A total of $500 \mu \mathrm{g}$ protein lysate from AS8B and AS1.6 were immunoprecipitated with anti-cyclin E was incubated with $50 \mu \mathrm{l}$ kinase buffer containing $5 \mu \mathrm{g}$ recombinant p27 and $10 \mu \mathrm{Ci}\left[\gamma_{-32}-\mathrm{P}\right] A T P$. Radio-labeled p27 was resolved by SDS-PAGE. (f) Increases of phosphorylated p27 in N-myc-expressing cells. A total of $500 \mu \mathrm{g}$ protein lysate from AS8B and AS1.6 were immunoprecipitated with anti-p27 was immunoblotted by anti-p27 and anti-T187 phosphorylated p27. (g) Increases of ubiquitinated p27 in Nmyc-expressing cells. A total of $750 \mu \mathrm{g}$ protein lysate from AS8B and AS1.6 were immunoprecipitated with anti-p27 and immunoblotted with anti-ubiquitin and anti-p27

total levels of p27 were decreased in the $\mathrm{N}$-myc-expressing cells (AS1.6), the relative levels of phospho-p27 increased by $50 \%$ in the $\mathrm{N}$-myc-expressing cells (Figure $4 \mathrm{e}$ ). Thus, consistent with the in vitro results in which $\mathrm{N}$-myc induced increases in cyclin $\mathrm{E}$ kinase activity and the ability of cyclin $\mathrm{E}$ kinase to phosphorylate p27, we find that there is more phosphorylated p27 in N-myc-expressing cells in vivo.
Since phosphorylation is necessary for p27 to be recognized by SCF complex and components of SCF (Skp1, Cul1, Skp2) are unchanged in N-myc-expressing cells, there should be an accumulation of ubiquitinated-p27 in N-myc-expressing cells. We investigated the ubiquitination of p27 by immunoprecipitation of p27 and immunoblotting for ubiquitin. The relative levels of poly-ubiquitinated p27 to total p27 were 
increased by $25 \%$ in $\mathrm{N}$-myc-expressing cells (Figure $4 \mathrm{~g}$ ). Taken together these results indicate that $\mathrm{N}$-myc affects the post-transcriptional regulation of p27 by stimulating an increase in cyclin $E$ levels and its kinase activity that in turn leads to increased levels of phosphorylated p27 and increases the pool of p27 to be ubiquitinated and degraded by the proteasome. $\mathrm{N}$-myc expression may also increase proteasome activity.

\section{RA decreases p27 degradation by decreasing ubiquitination of $\mathrm{p} 27$ in KCNR cells}

After finding that $\mathrm{N}$-myc overexpression led to a decrease in p27 levels, we sought to study how the RA induced decrease in N-myc levels would affect p27 levels. Since N-myc increases the degradation activity of p27, the downregulation of $\mathrm{N}$-myc by RA treatment should induce a decrease in p27 degradation resulting in an increase in p27 levels. To examine whether RA affects degradation of p27, an in vitro p27 degradation assay was performed. In untreated cells it took $2.5 \mathrm{~h}$ to degrade $50 \%$ of His- p27 while in RA-treated cells in which $\mathrm{N}$-myc levels were decreased it took almost $7 \mathrm{~h}$ to degrade $50 \%$ of His-p27 (Figure $5 a$ ).

$\mathrm{N}$-myc stimulates cyclin E-dependent kinase activity leading to increased phosphorylation of p27. Since RA decreases $\mathrm{N}$-myc levels followed by a decrease in cyclin E-dependent kinase activity (Figure 1$)^{10}$ there should be a decrease in phosphorylation of p27 in RA-treated cells. To examine this, p27 was immunoprecipitated from control and RA-treated cells, resolved by SDS-PAGE analysis and immunoblotted with antiphosphorylated p27 antibody. Phosphorylated p27 levels decrease even though total p27 levels increase in RAtreated cells (Figure $5 \mathrm{~b}$ ). The ratio of phosphorylated p27 to total p27 was decreased by $65 \%$ in RA-treated cells.

Although N-myc overexpression does not alter the expression of SCF components (Figure 4d), it is possible that RA alters other molecules regulating the targeting of p27 for degradation. We assessed the steady-state levels of SCF components including Skp1, Cul1, and Skp2. Interestingly, the steady-state levels of Skp2 was decreased by RA treatment in a time-dependent manner while Skp1 and Cul1 were not significantly changed (Figure 5c). In the same samples we analysed the kinetics of p27 and N-myc by Western blot analysis. Levels of p27 increased in a timedependent manner as the levels of $\mathrm{N}$-myc decreased (Figure $5 c$ ). Densitometric analysis of the steady-state levels of p27 indicated that there is a bimodal increase in p27 levels, an early phase before $48 \mathrm{~h}$ and a late phase after $48 \mathrm{~h}$ (Figure $5 \mathrm{c}$, lower panel). The decrease in N-myc levels precedes the accumulation of $\mathrm{p} 27$ that is first detected at $24 \mathrm{~h}$ and coincides with the decrease in phosphorylated p27. Although Skp2 levels did not decrease within the first $24 \mathrm{~h}$ there was an almost $80 \%$ decrease in Skp2 levels at $72 \mathrm{~h}$ after RA treatment (Figure 5c). The decrease in Skp2 levels temporally precedes the second increase in p27 levels (Figure 5c, lower panel). These results suggest that RA differentially regulates $\mathrm{N}$-myc and Skp2. Our finding that N-myc overexpression did not affect Skp2 levels (Figure 4c) indicates that the decrease in Skp2 levels by RA is independent of $\mathrm{N}$-myc. Thus there are at least two mechanisms by which RA regulates the targeting of p27 for degradation, one by downregulating N-myc and the cyclin E-dependent kinase mediated phosphorylation of p27 and the other via the N-myc-independent decrease of Skp2 levels.

Phosphorylated p27 can be recognized by Skp2, ubiquitinated and targeted for degradation by the proteasome. ${ }^{27}$ To investigate the role of the proteasome in modulating p27 levels during RA induced growth control of NB cells, we treated KCNR cells with RA in the presence of LC. In control cells LC treatment leads to a two-fold increase in p27 levels. However, in RA-treated cells in which the steady-state p27 levels were three-fold higher than control cells, treatment with LC did not lead to a dramatic increase in p27 levels (Figure $5 d)$. This result suggests that in control cells the proteasome contributes to maintaining the steady-state levels of p27 in NB cells while in RA-treated cells the proteasome does not markedly affect p27 levels. To determine the effect of RA on the proteasome, we assessed the in vitro proteasome activity in KCNR cells treated with RA for $72 \mathrm{~h}$. There was no significant change in proteasome activity between RA-treated cells and control cells (Figure $5 \mathrm{e}$ ). These results indicate that the activity of the proteasome per se is not altered by RA and suggest mechanisms that target p27 for degradation are critical for the regulation of p27 in RA-treated NB cells.

We propose that the decrease in Skp2 levels and the decrease of p27 phosphorylation lead to a decrease in the ubiquitination of p27 in RA-treated cells. To address this hypothesis, p27 was immunoprecipitated protein lysate from KCNR cells treated with RA for $72 \mathrm{~h}$ and followed by Western blot analysis for ubiquitin. Although total p27 levels increased in RA-treated cells, levels in ubiquitinated p27 slightly decreased. Thus, the relative levels of polyubiquitinated p27 to total p27 were decreased by $85 \%$ (Figure $5 f$ ). These results indicate that RA regulates p27 levels by decreasing the targeting of $\mathrm{p} 27$ for the ubiquitin-proteasome pathway rather than through degradation by the $26 \mathrm{~S}$ proteasome. In N-mycexpressing cells the RA induced changes in the targeting of p27 to the proteasome are mediated by the decreased levels of N-myc and Skp2

\section{Discussion}

In this study, we examined the mechanism(s) by which retinoids regulated the cell cycle machinery and how N-myc overexpression affected both the growth promoting as well as the growth inhibiting aspects of cell cycle progression. Key to both the ability of RA to arrest NB cell growth and for N-myc to stimulate cell cycle was the regulation of p27 expression. We found that N-myc overexpression decreases p27 mRNA and increases p27 degradation via cyclin $E$ kinase-dependent phosphorylation of p27 as well as increases in proteasome activity. We identified two mechanisms that regulate the targeting of $\mathrm{p} 27$ for degradation during RA induced $\mathrm{G} 1$ arrest in N-myc amplified NB cells (1) an N-myc-dependent decrease in phosphorylation of p27 and (2) an N-mycindependent decrease in Skp2 protein levels. The activity of the proteasome per se was not altered by RA treatment. 
a

b

C

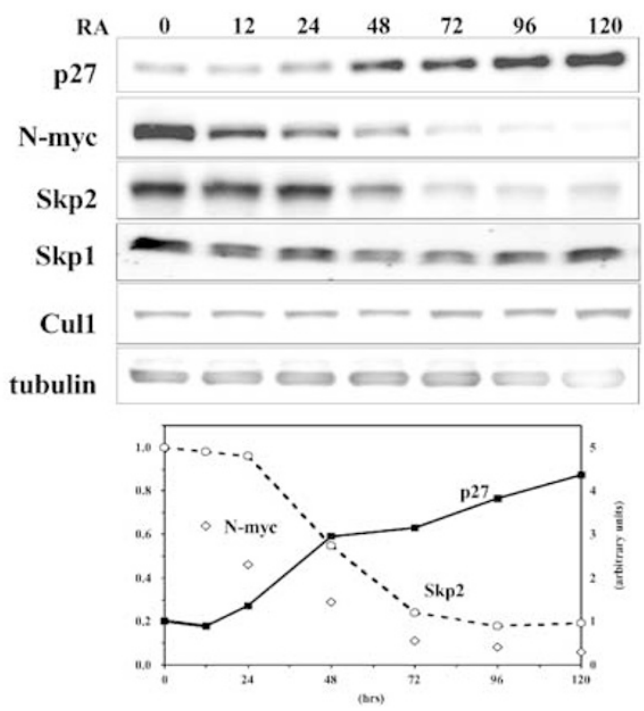

d

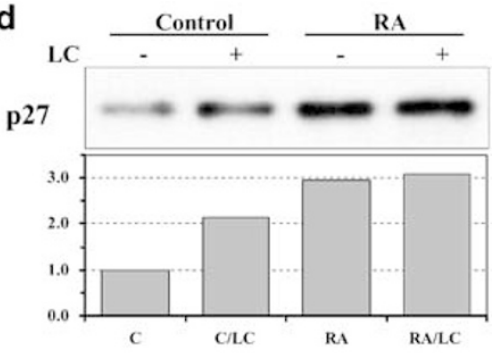

(hrs)

e

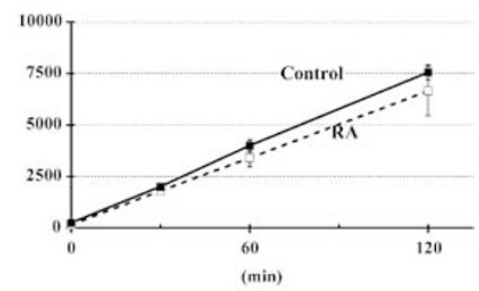

f

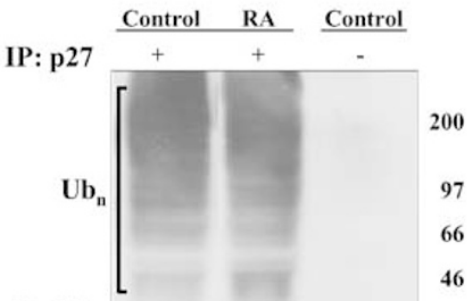

IB: Ub

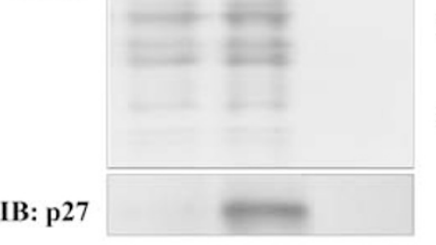

30

21

$\mathrm{Ub}_{\mathrm{n}} / \mathrm{p} 27 \quad \underline{1.00} \quad 0.15$

Figure 5 RA decreases p27 degradation by decreasing ubiquitination of p27 in KCNR cells. (a) p27 in vitro degradation assay. His-tagged recombinant p27 were incubated with cell extracts (S100) from KCNR cells treated with or without RA for $72 \mathrm{~h}$ for indicated time and his-tagged p27 was detected by immunoblotting using antiHis antibody. (b) RA decreases phosphorylated p27. A total of $500 \mu \mathrm{g}$ protein lysate KCNR cells treated with or without RA for $72 \mathrm{~h}$ were immunoprecipitated with antip27 and immunoblotted by anti-p27 and anti-T187 phosphorylated p27. (c) Kinetics analysis for p27, N-myc and SCF components (Skp1, Cul1, Skp2). KCNR cells were treated with RA for various time and protein lysates were analyzed by Western blot by appropriate antibodies. Densitometric analysis were performed for p27, $\mathrm{N}$-myc and Skp2 kinetics. (d) Inhibition proteasome does not increase p27 in RA-treated cells. After $72 \mathrm{~h}$ RA treatment, KCNR cells were treated with $2 \mu \mathrm{M} \mathrm{LC}$ for $12 \mathrm{~h}$ before harvest and total protein was subjected for Western blot analysis for N-myc and 27. (e) RA does not affect proteasome activity. Total protein lysates from KCNR cells treated with or without RA were subjected for in vitro proteasome activity assay. (f) Decreases of ubiquitinated p27 in RA-treated cells. A total of $750 \mu \mathrm{g}$ protein lysate from KCNR cells treated with or without RA were immunoprecipitated with anti-p27 and immunoblotted with anti-ubiquitin and anti-p27.

p27 plays an important role in both cell proliferation and cell differentiation. ${ }^{17,28}$ While p27 is a necessary component of cyclin D-dependent kinase activity, high levels of p27 inhibit cyclin D- and E-dependent kinase activity. High levels of p27 also stimulated differentiation in some model systems ${ }^{22,32}$ and apoptosis in others. ${ }^{33}$ Since increased p27 levels by adenovirus infection induced cell cycle arrest with minimal cell death in NB cells, we focused our studies on how p27 affects the cell cycle during the RA induced G1 arrest of NB cells
(Figure 2b). While the ability of c-myc to stimulate cell cycle progression and alter the cell cycle machinery has been intensely studied, ${ }^{7-10}$ analogous studies have not been preformed on N-myc and L-myc. Even though N-myc is able to functionally complement c-myc during development, ${ }^{11,13}$ not all c-myctarget genes are expressed in $\mathrm{N}$-myc-expressing NB cells. ${ }^{34}$ For this reason, we felt it was important to study how N-myc affected the cell cycle machinery in NB cells. Our finding that $\mathrm{N}$-myc stimulated cyclin $\mathrm{E}$ and cyclin 
E-dependent kinase activity and increased E2F-1 mRNA levels were consistent with Leone et al.,${ }^{35}$ who showed that in ras-activated cells, c-myc stimulates cyclin E-dependent kinase activity and E2F. Although c-myc has been shown to stimulate cyclin $\mathrm{D}$ expression in normal cells, ${ }^{9}$ we failed to detect increases in any member of the cyclin $D$ family when $\mathrm{N}$ myc was overexpressed in the AS NB cells.

In our model, we showed that N-myc altered p27 levels and there was an inverse correlation between N-myc and p27 expression in KCNR cells treated with RA (Figure 5c). During the cell cycle p27 levels are mainly modulated at the posttranscriptional level ${ }^{24}$ via degradation of p27 by the ubiquitinproteasome pathway ${ }^{25}$ and/or the sequestration of $\mathrm{p} 27$ by c$m y c$ via increases in the levels of cyclin D/Cdk kinases. N-myc overexpression stimulates cyclin E, E2F-1 expression, and cyclin E-dependent kinase activity while decreasing p27 levels (Figure 3). Cyclin E-dependent kinases are known to phosphorylate threonine-187 of p27 and target it to the ubiquitination to be degraded by $26 \mathrm{~S}$ proteasome. ${ }^{25,31}$ In fact, $\mathrm{N}$-myc-expressing cells have increased cyclin E-dependent kinase activity as demonstrated in vitro using recombinant p27 as the substrate (Figure 4e). Thus our results are consistent with a model in which $\mathrm{N}$-myc stimulates increases in cyclin E-dependent kinase activity that in turn leads to increased phosphorylation of p27 and degradation. Consistent with this finding, we detected more phosphorylated p27 in $\mathrm{N}$-myc-transfected cells than in control-transfected cells (Figure 4f) and the rate of p27 degradation and proteasome activity were higher in $\mathrm{N}$-myc-transfected cells than controltransfected cells (Figure 4a). A recent study using Serial Analysis of Gene Expression (SAGE) showed that proteasome subunit 6 ( $\beta$ type), subunit $\beta$ (type 7 ), and subunit $\alpha$ (type 7) were increased in N-myc-expressing NB cells, ${ }^{36}$ which may affect proteasome function.

In RA-treated N-myc amplified NB cells there was a bimodal increase in p27 levels. RA decreased N-myc levels and this was accompanied by decreased levels of phosphorylated p27 despite increased total p27 levels (Figure 5b). We also found that while the rate of $p 27$ degradation was reduced (Figure $5 a)$, the activity of the proteasome was not affected in RAtreated cells (Figure $5 \mathrm{e}$ ). These results suggested that RA regulated $\mathrm{p} 27$ at the level of targeting it for degradation rather than at the level of degradation by the proteasome. Borriello et al. $^{37}$ suggested that RA-treated NB cells lack some component(s) of p27 proteolytic pathway and that N-myc is not directly involved in pathways controlling p27 levels because the accumulation of p27 in RA-treated NB cells occurred regardless of the expression of the $\mathrm{N}$-myc gene. We propose that there are at least two mechanisms that affect p27 levels in RA-treated N-myc-expressing NB cells - an N-myc-dependent and -independent mechanism. The decrease in N-myc levels was coincident with the early phase of the p27 accumulation detected in RA-treated cells (Figure $5 \mathrm{c}$ ). This mechanism could account for the ability of constitutive N-myc to block RA induced growth control ${ }^{14}$ or the findings of increased $\mathrm{N}$-myc expression and growth in RA-resistant cells. ${ }^{38}$ It is known that Skp2 is a key component in the regulation of p27 levels by mediating its ubiquitination. ${ }^{27}$ The steady-state levels of Skp2 decreased coincident with the late phase increase in total p27 in RA-treated KCNR cells (Figure
$5 a)$, and was not significantly different in N-myc overexpressing cells (Figure $4 d$ ). We propose that Skp2 is the N-mycindependent component of the p27 ubiquitin degradation path missing in RA-treated NB cells. Other genes implicated in post-translational regulation of p27, the c-myc target gene Cul1, the Cdk subunit 1 (Cks1) and Jab1 were unchanged in RA-treated cells or in cells differentially expressing N-myc (data not shown).

In addition to p27 degradation by ubiquitin-proteasome pathway, there was another possible post-transcriptional mechanism, which may contribute to p27 levels. We found that a decrease in Cdk6 levels was associated with RAinduced G1 arrest (Figure 1c). Interestingly RA altered only Cdk6 expression but not Cdk2 and 4. The downregulation of Cdk6 by RA could contribute to the increase in p27 levels because p27 binds to cyclin D/Cdk6 complexes in cycling cells. The RA induced decrease in Cdk6 may release p27 from cyclin D/Cdk6 complexes. In control cells lysates immunodepletion of cyclin D1 removes all p27, however, p27 remained in RA-treated cells after immunodepletion of cyclin D1 (data not shown).

As retinoids are currently used in the treatment of advance stage NB tumors, it is likely that RA-resistant cells will arise. This study forms a basis to investigate mechanisms of retinoid resistance that may involve alterations in cell cycle proteins. Selection for RA-resistant NB cells identified several cell lines that continue to cycle in the presence of $\mathrm{RA}^{39}$ and have mutations in the RA regulatory region of the $\mathrm{N}$-myc promoter that mediates downregulation of $\mathrm{N}$-myc. Recently, Bergmann et al. ${ }^{16}$ found that p27 is a good prognostic factor in NB yet this was independent of N-myc amplification. Our model indicates that p27 levels are dependent on the levels of $\mathrm{N}$-myc protein expression. While amplified $\mathrm{N}$-myc tumors have high levels of $\mathrm{N}$-myc mRNA and protein, single-copy NB cells and tumors may also have high levels of $\mathrm{N}$-myc protein. High levels of $\mathrm{N}$-myc protein or mRNA expression predict an unfavorable prognosis in NB regardless of $\mathrm{N}$-myc gene amplification. In future studies, we would like to evaluate whether p27, and N-myc expression would have dependent or independent prognostic value. Since our findings indicate $\mathrm{N}$-myc stimulates cyclin $\mathrm{E}$ and E2F and increases in phosphorylated -p27, we would like to evaluate whether these proteins may be overexpressed in poor prognosis tumors and if they have prognostic significance.

Our studies have identified two important aspects of targeting of p27 for degradation that may be of therapeutic value. Potentially one might block the ability of p27 to be phosphorylated perhaps via cyclin $\mathrm{E}$ kinase inhibitors or a small molecule that blocks the recognition of phosphorylated p27 by SKP2 and prevents p27 degradation. Such treatments alone or in combination with retinoids would increase p27 levels and arrest cell growth.

\section{Materials and Methods}

\section{RA}

All-trans RA (SIGMA, St Louis, MO, USA) is suspended in 95\% ethanol and stored at $-20^{\circ} \mathrm{C}$ for a month protected from light. 


\section{Cell culture}

The SMS-KCNR cell line contains approximately 30-50 copies of MYCN and expresses relatively high levels of $\mathrm{N}$-myc with little to no detectable $\mathrm{c}$ myc expression. The SK-N-AS cell line contains a single copy of MYCN and expresses little to no detectable $\mathrm{N}$-myc but expresses c-myc. Cell lines were cultured in RPMI supplemented with $10 \%$ fetal bovine serum and after $24 \mathrm{~h}$, KCNR cells were treated with $5 \mu \mathrm{M}$ all-trans RA for an additional $72 \mathrm{~h}$. SK-N-AS cell lines transfected with control vector $(6 \mathrm{~B}, 8 \mathrm{~B})$ or $\mathrm{N}-\mathrm{myc}$ $(1.6,14.2)$ were cultured in RPMI supplemented with $10 \%$ fetal bovine serum containing $300 \mu \mathrm{g} / \mathrm{ml} \mathrm{G} 418 .^{29}$

\section{Antibodies and reagents}

Anti-cyclin D (H-295), anti-cyclin E (C-19-G), anti-Cdk2 (M2-G), anti-Cdk4 (C-22-G), anti-Cdk6 (C-21-G), anti-p27 (C-19), anti-E2F-1 (C-20), anti-Nmyc (C-19), anti-Skp1 (H-1630), anti-Skp2 (H-435), anti-ubiquitin (P4D1), and anti-His-probe $(\mathrm{H}-15)$ were obtained from Santa Cruz Biotech (Santa Cruz, CA, USA). Anti-cyclin D1 was obtained from CLONTECH (Palo Alto, CA, USA). Anti-p21 (Ab-5) and anti- $\alpha$ tubulin were obtained from Oncogene Science (Cambridge, MA, USA). Anti-T187 phosphorylatedp27 and anti-Cul1 were obtained from ZYMED (S San Francisco, CA, USA). Each antibody was used according to the manufacturer's recommendations.

\section{BrdU incorporation assay}

KCNR cells treated with or without RA for $72 \mathrm{~h}$ were pulse-labeled with $10 \mu \mathrm{M}$ BrdU for 30 min and the incorporated BrdU into DNA was identified by anti-BrdU antibody (Becton Dickinson, San Jose, CA, USA) using flow cytometry (Becton Dickinson, San Jose, CA, USA). The protocol was performed according to the manufacturer's specifications.

\section{p27 adenovirus infection}

$\mathrm{KCNR}$ cells were pretreated with $1 \mathrm{nM} \mathrm{RA}$ at $24 \mathrm{~h}$ prior to virus exposure to increase the efficiency of adenovirus infection. Cells were treated with viruses, p27-Ad and null-Ad. ${ }^{33}$ After $24 \mathrm{~h}$, cells were washed and cultured for an additional $72 \mathrm{~h}$.

\section{RNA analysis}

For Northern blot analysis, total RNA was isolated using an RNAeasy Kit (Quiagen, Santa Clarita, CA, USA) and $30 \mu \mathrm{g}$ total RNA was used for Northern blotting as described previously. ${ }^{6}$ The cDNA fragment of Cdk6 (a gift from Dr. E Harlow, UK), Cdk2, Cdk4, and GAPDH were used as probes.

\section{Protein analysis}

For Western blot analysis, $20-40 \mu \mathrm{g}$ of protein lysate/sample were electrophoresed on Tris-Glycine gels and transferred to $0.2 \mu \mathrm{m}$ pore nitrocellulose membranes. Membranes were incubated with the appropriate primary antibody, washed and incubated with the appropriate horse radish peroxidase (HRP)-conjugated secondary antibody. Finally, the HRP-conjugated antibody was detected using SuperSignal substrates (PIERCE, Rockford, IL, USA). For immunoprecipitation, $500 \mu \mathrm{g}$ of protein lysate were incubated with Protein G PLUS-Agarose, Protein A-Agarose (Santa Cruz, CA, USA) for $30 \mathrm{~min}$ at $4^{\circ} \mathrm{C}$ to preclear then immunoprecipitated with the indicated antibody for $1 \mathrm{~h}$ at $4^{\circ} \mathrm{C}$. The immunoprecipita- tion was washed with protein lysis buffer for use in immunoblotting and in vitro kinase analysis. For in vitro kinase assays, the washed immunoprecipitated beads were incubated with kinase buffer containing histone $\mathrm{H} 1$ (Boehringer Mannheim, Indianapolis, IN, USA) or recombinant p27 (a gift from Dr. M Pagano) and $10 \mu \mathrm{Ci}\left[\gamma^{32} \mathrm{P}\right]$ ATP at $30^{\circ} \mathrm{C}$ for $30 \mathrm{~min}$ to determine the kinase activity of cyclin $E$ and Cdk2. Cyclin $D$ kinase assays were performed using a GST-Rb fusion protein (Santa Cruz Biotech, Santa Cruz, CA, USA) as a substrate. The radiolabeled product was resolved by SDS-PAGE. Proteasome activity assays were carried out according to the procedure of Lightcap et al. ${ }^{30}$ Soluble protein of $5-25 \mu \mathrm{g}$ was incubated at $30^{\circ} \mathrm{C}$ in a buffer consisting of $20 \mathrm{mM} \mathrm{pH} 8.0 \mathrm{HEPES}$, 0.5 mM EDTA, 60 uM Suc-LLVY-AMC (Biomol, Plymouth Meeting, PA, USA), $1 \%$ DMSO, and $0.035 \%$ SDS. The fluorescent signal generated by the release of AMC was measured by a Victor2 1420 Multilabel Counter (Wallac, Gaithersburg, MD, USA). For the in vitro degradation assay, the cell pellets were resuspended in hypotonic buffer $(50 \mathrm{mM}$ Tris- $\mathrm{HCl}, \mathrm{pH} 8.0$, $5 \mathrm{mM} \mathrm{MgCl} 2,1 \mathrm{mM} \mathrm{DTT}, 2 \mathrm{mM} \mathrm{ATP}$ ), frozen and thawed three times followed by centrifugation at $100000 \times g$ for $1 \mathrm{~h}$ at $4^{\circ} \mathrm{C}$. The supernatant $(\mathrm{S} 100)$ was stored at $-80^{\circ} \mathrm{C}$. His-tagged p27 $(0.1 \mu \mathrm{g})$ was incubated with $50 \mu \mathrm{g}$ of the $\mathrm{S} 100$ cell extracts for 0,1 , and $3 \mathrm{~h}$ in $30 \mu \mathrm{l}$ of the ATPregeneration buffer $\left(50 \mathrm{mM}\right.$ Tris- $\mathrm{HCl}, \mathrm{pH} 8.0,5 \mathrm{mM} \mathrm{MgCl}_{2}, 1 \mathrm{mM}$ DTT, $2 \mathrm{mM} \mathrm{ATP}, 100 \mu \mathrm{g} / \mathrm{ml}$ creatin phosphokinase, $10 \mathrm{mM}$ phosphocreatine). Proteins were resolved by SDS-PAGE and detected by blotting with antiHis antibody. Recombinant His-tagged p27 was a gift from Dr M Pagano.

\section{Acknowledgments}

We wish to thank Dr. M Pagano for recombinant His-tagged p27, Dr. E Harlow for cdk6 and Dr. P Seth for null and p27 containing adenovirus.

\section{References}

1. Schwartz AD, Dadash-Zadeh M, Lee H and Swaney JJ (1974) Spontaneous regression of disseminated neuroblastoma. J. Pediatr. 85: 760-763

2. Brodeur GM, Seeger RC, Schwab M, Varmus HE and Bishop JM (1984) Amplification of $\mathrm{N}$-myc in untreated human neuroblastomas correlates with advanced disease stage. Science 224: 1121-1123

3. Thiele CJ, Reynolds CP and Israel MA (1985) Decreased expression of N-myc precedes retinoic acid-induced morphological differentiation of human neuroblastoma. Nature 313: 404-406

4. Matthay KK, Villablanca JG, Seeger RC, Stram DO, Harris RE, Ramsay NK, Swift P, Shimada H, Black CT, Brodeur GM, Gerbing RB and Reynolds CP (1999) Treatment of high-risk neuroblastoma with intensive chemotherapy, radiotherapy, autologous bone marrow transplantation, and 13-cis-retinoic acid. Children's Cancer Group. N. Engl. J. Med. 341: 1165-1173

5. Sidell N (1982) Retinoic acid-induced growth inhibition and morphologic differentiation of human neuroblastoma cells in vitro. J. Natl. Cancer Inst. 68: 589-596

6. Matsuo T and Thiele CJ (1998) p27Kip1: a key mediator of retinoic acid induced growth arrest in the SMS-KCNR human neuroblastoma cell line. Oncogene 16: 3337-3343

7. Rudolph B, Saffrich R, Zwicker J, Henglein B, Muller R, Ansorge W and Eilers $M$ (1996) Activation of cyclin-dependent kinases by Myc mediates induction of cyclin A, but not apoptosis. EMBO J. 15: 3065-3076

8. Steiner P, Philipp A, Lukas J, Godden-Kent D, Pagano M, Mittnacht S, Bartek J and Eislers M (1995) Identification of a Myc-dependent step during the formation of active G1 cyclin-cdk complexes. EMBO J. 14: 4814-4826

9. Vlach J, Hennecke S, Alevizopoulos K, Conti D and Amati B (1996) Growth arrest by the cyclin-dependent kinase inhibitor p27Kip1 is abrogated by c-Myc. EMBO J. 15: 6595-6604

10. Bouchard C, Thieke K, Maier A, Saffrich R, Hanley-Hyde J, Ansorge W, Reed S, Sicinski P, Bartek J and Eilers M (1999) Direct induction of cyclin D2 by Myc 
contributes to cell cycle progression and sequestration of p27. EMBO J. 18 : $5321-5333$

11. Malynn BA, de Alboran IM, O'Hagan RC, Bronson R, Davidson L, DePinho RA and Alt FW (2000) N-myc can functionally replace c-myc in murine development, cellular growth, and differentiation. Genes Dev. 14: 1390-1399

12. Lutz W, Stohr M, Schurmann J, Wenzel A, Lohr A and Schwab M (1996) Conditional expression of $\mathrm{N}$-myc in human neuroblastoma cells increases expression of alpha-prothymosin and ornithine decarboxylase and accelerates progression into S-phase early after mitogenic stimulation of quiescent cells. Oncogene 13: 803-812

13. Aubry S and Charron J (2000) N-Myc shares cellular functions with c-Myc. DNA Cell Biol. 19: 353-364

14. Peverali FA, Orioli D, Tonon L, Ciana P, Bunone G, Negri M and Della-Valle G (1996) Retinoic acid-induced growth arrest and differentiation of neuroblastoma cells are counteracted by $\mathrm{N}$-myc and enhanced by max overexpressions. Oncogene 12: 457-462

15. Lasorella A, Noseda M, Beyna M, Yokota $Y$ and lavarone $A(2000)$ Id2 is a retinoblastoma protein target and mediates signaling by Myc oncoproteins. Nature (Lond.) 407: 592-598

16. Bergmann E, Wanzel M, Weber A, Shin I, Christiansen H and Eilers M (2001) Expression of P27(KIP1) is prognostic and independent of MYCN amplification in human neuroblastoma. Int. J. Cancer 95: 176-183

17. Lloyd RV, Erickson LA, Jin L, Kulig E, Qian X, Cheville JC and Scheithauer BW (1999) p27kip1: a multifunctional cyclin-dependent kinase inhibitor with prognostic significance in human cancers. Am. J. Pathol. 154: 313-323

18. Sherr CJ (1993) Mammalian G1 cyclins. Cell. 18: 1059-1065

19. LaBaer J, Garrett MD, Stevenson LF, Slingerland JM, Sandhu C, Chou HS, Fattaey A and Harlow E (1997) New functional activities for the p21 family of CDK inhibitors. Genes Dev. 11: 847-862

20. Sherr CJ and Roberts JM (1999) CDK inhibitors: positive and negative regulators of G1-phase progression. Genes Dev. 13: 1501-1512

21. Sherr CJ (2000) The Pezcoller lecture: cancer cell cycles revisited. Cancer Res. 60: 3689-3695

22. Wang QM, Jones JB and Studzinski GP (1996) Cyclin-dependent kinase inhibitor p27 as a mediator of the G1-S phase block induced by 1,25 dihydroxyvitamin D3 in HL60 cells. Cancer Res. 56: 264-267

23. Durand B, Gao FB and Raff M (1997) Accumulation of the cyclin-dependent kinase inhibitor p27/Kip1 and the timing of oligodendrocyte differentiation. EMBO J. 16: 306-317

24. Hengst $L$ and Reed SI (1996) Translational control of p27Kip1 accumulation during the cell cycle. Science 271: 1861-1864

25. Pagano M, Tam SW, Theodoras AM, Beer-Romero P, Del Sal G, Chau V, Yew PR and Draetta GF (1995) Role of the ubiquitin-proteasome pathway in regulating abundance of the cyclin-dependent kinase inhibitor p27. Science 269: 682-685

26. Montagnoli A, Fiore F, Eytan E, Carrano AC, Draetta GF, Hershko A and Pagano M (1999) Ubiquitination of p27 is regulated by Cdkdependent phosphorylation and trimeric complex formation. Genes Dev. 13: $1181-1189$
27. Carrano AC, Eytan E, Hershko $A$ and Pagano M (1999) SKP2 is required for ubiquitin-mediated degradation of the CDK inhibitor p27. Nat. Cell Biol. 1: 193199

28. Sgambato A, Cittadini A, Faraglia B and Weinstein IB (2000) Multiple functions of p27 (Kip1) and its alterations in tumor cells: a review. J. Cell. Physiol. 183: $18-27$

29. Feltner DE, Cooper M, Weber J, Israel MA and Thiele CJ (1989) Expression of class I histocompatibility antigens in neuroectodermal tumors is independent of the expression of a transfected neuroblastoma myc gene. J. Immunol. 143: 4292-4299

30. Lightcap ES, McCormack TA, Pien CS, Chau V, Adams J and Elliott PJ (2000) Proteasome inhibition measurements: clinical application. Clin. Chem. 46: 673-683

31. Vlach J, Hennecke S and Amati B (1997) Phosphorylation-dependent degradation of the cyclin-dependent kinase inhibitor p27. EMBO J. 16: 5334-5344

32. Drissi H, Hushka D, Aslam F, Nguyen Q, Buffone E, Koff A, van Wijnen A, Lian JB, Stein JL and Stein GS (1999) The cell cycle regulator p27kip1 contributes to growth and differentiation of osteoblasts. Cancer Res. 59: 3705-3711

33. Craig C, Wersto R, Kim M, Ohri E, Li Z, Katayose D, Lee SJ, Trepel J, Cowan K and Seth $P$ (1997) A recombinant adenovirus expressing p27Kip1 induces cell cycle arrest and loss of cyclin-Cdk activity in human breast cancer cells. Oncogene 14: 2283-2289

34. Ben-Yosef T, Yanuka O, Halle D and Benvenisty N (1998) Involvement of Myc targets in c-myc and N-myc induced human tumors. Oncogene 17: 165-171

35. Leone G, DeGregori J, Sears R, Jakoi L and Nevins JR (1997) Myc and Ras collaborate in inducing accumulation of active cyclin E/Cdk2 and E2F. Nature. 387: 422-426

36. Boon $\mathrm{K}$, Caron $\mathrm{HN}$, van Asperen $\mathrm{R}$, Valentijn $\mathrm{L}$, Hermus $M C$, van Sluis $\mathrm{P}$, Roobeek I, Weis I, Voute PA, Schwab M and Versteeg R (2001) N-myc enhances the expression of a large set of genes functioning in ribosome biogenesis and protein synthesis. EMBO J. 20: 1383-1393

37. Borriello A, Pietra VD, Criscuolo M, Oliva A, Tonini GP, Iolascon A, Zappia V and Ragione FD (2000) p27Kip1 accumulation is associated with retinoicinduced neuroblastoma differentiation: evidence of a decreased proteasomedependent degradation. Oncogene 19: 51-60

38. Amati B, Alevizopoulos K and Vlach J (1998) Myc and the cell cycle. Front. Biosci. 3: D250-D268

39. Matsumoto K, Wada RK, Yamashiro JM, Kaplan DR and Thiele CJ (1994) Signals transducted via insulin-like growth factor I receptor (IGFR) mediate resistance to retinoic acid-induced cell growth arrest in a human neuroblastoma cell line. Cell Death Differ. 1: 49-58

40. Hong CM, Sidell N, Tuthill MC and Wada RK (2000) Identification of retinoic acid (R4) response elements in N-myc and correlation of promoter mutation with RA-resistance of neuroblastoma. Proc. Amer. Assoc. Cancer Res. 41: 112.

41. Hiyama E, Hiyama K, Yokoyama T and Ishii T (1991) Immunohistochemical analysis of $\mathrm{N}$-myc protein expression in neuroblastoma: correlation with prognosis of patients. J. Pediatr. Surg. 26: 838-843 